\title{
Irinotecan, docetaxel and oxaliplatin combination in metastatic gastric or gastroesophageal junction adenocarcinoma
}

\author{
L Di Lauro*,', C Nunziata ${ }^{2}$, MG Arena ${ }^{3}$, P Foggi', I Sperduti ${ }^{4}$ and M Lopez' \\ 'Division of Medical Oncology B, 'Regina Elena' Institute for Cancer Research, Via Elio Chianesi, 53, Rome 00 I 44, Italy; ${ }^{2}$ Division of Medical Oncology, \\ San Giuseppe Hospital, Albano Laziale 0004I, Italy; ${ }^{3}$ Division of Medical Oncology, Toraldo Hospital, Tropea 88038, Italy; ${ }^{4}$ Biostatistics Unit, 'Regina \\ Elena' Institute for Cancer Research, Rome 00 I44, Italy
}

\begin{abstract}
This phase II study was designed to evaluate the activity and safety of a combination of irinotecan, docetaxel and oxaliplatin in metastatic gastric or gastroesophageal junction (GEJ) adenocarcinoma. Forty patients with measurable distant metastasis received irinotecan $150 \mathrm{mg} \mathrm{m}^{-2}$ and docetaxel $60 \mathrm{mg} \mathrm{m}^{-2}$ on day I, and oxaliplatin $85 \mathrm{mg} \mathrm{m}^{-2}$ on day 2. Cycles were repeated every 3 weeks. The primary end point was to demonstrate a $50 \%$ improvement in time-to-progression (TTP) over historical controls. All patients were evaluable. Median TTP was 6.5 months (95\% confidence interval (Cl) 5.6-7.4), the overall response rate was 50\% (95\% Cl 35$65 \%$ ) and the median overall survival was 11.5 months (95\% Cl 8.7-14.3). Grade 3/4 neutropaenia occurred in $47.5 \%$ of patients. There were four episodes of febrile neutropaenia in three patients. Other non-haematological grade 3 toxicities included diarrhoea in four patients (10\%), vomiting in three patients (7.5\%) and mucositis in two patients (5\%). The irinotecan, docetaxel and oxaliplatin combination chemotherapy is an active and well-tolerated novel regimen for treating metastatic gastric or GEJ adenocarcinoma and deserves further evaluation in randomised trials and in combination with molecular targeting agents.

British Journal of Cancer (2007) 97, 593-597. doi: I0.1038/sj.bjc.66039I7 www.bjcancer.com
\end{abstract}

Published online 31 July 2007

(c) 2007 Cancer Research UK

Keywords: docetaxel; irinotecan; metastatic gastric cancer; oxaliplatin

Gastric cancer is still one of the most prevalent human malignancies, and remains the second leading cause of cancer mortality in the world (Kamangar et al, 2006). While the incidence of distal tumours is declining in the Western countries, the incidence of gastroesophageal junction (GEJ) adenocarcinomas, which appear to be more virulent, is rapidly increasing (Devesa and Fraumeni, 1999). The outcome among patients with gastric cancer is determined by the stage of the disease at presentation. Generally, a potentially curative resection is possible in only $30 \%$ of all recently diagnosed patients (Hundahl et al, 2000), since in the majority of cases the disease is locally advanced or metastatic. Systemic chemotherapy is often used in these patients because randomised trials have shown that combination regimens confer a survival advantage and an improvement in quality-of-life when compared with supportive care alone. Overall, the results have been mostly unsatisfactory with a median time-to-progression (TTP) of 4 months and a median overall survival (OS) of 8-9 months (Wagner et al, 2006).

During the last few years, several new drugs including irinotecan (Bleiberg, 1999; Kohne et al, 1999), oxaliplatin (Louvet et al, 2002; Al-Batran et al, 2004), capecitabine (Ajani, 2006) and docetaxel (Thuss-Patience et al, 2006) have been demonstrated to be active in metastatic gastric cancer. The incorporation of these agents in new combinations has resulted in regimens such as DCF (docetaxel, cisplatin, fluorouracil), EOX (epirubicin, oxaliplatin, capecitabine) and ILF (irinotecan, leucovorin, fluorouracil), which

*Correspondence: Dr L Di Lauro; E-mail: dilauro@ifo.it

Received 18 May 2007; revised 4 July 2007; accepted 9 July 2007; published online 31 July 2007 could have a role in the treatment of these patients but survival is still very poor (Rivera et al, 2007). Recently, the combination of irinotecan and oxaliplatin showed activity in advanced gastric cancer with a $50 \%$ response rate (RR) and a favourable toxicity profile in 32 patients (Souglakos et al, 2004). Furthermore, a synergic effect has been reported with the combination of docetaxel and irinotecan both in untreated and treated patients with gastric cancer (Chang et al, 2003; Hawkins et al, 2003). It seemed thus appropriate to test the activity and toxicity of three new agents in combination, namely irinotecan, docetaxel and oxaliplatin (IDO), in patients with metastatic gastric or GEJ cancer. These agents have distinct and complementary mechanisms of action, they lack cross-resistance, and they have favourable toxicity profiles. To this purpose, we first performed a phase I study of this combination according to the modified Fibonacci schema. Six patients entered this study, and the recommended phase II doses were as follows: irinotecan $150 \mathrm{mg} \mathrm{m}^{-2}$ and docetaxel $60 \mathrm{mg} \mathrm{m}^{-2}$ on day 1 , oxaliplatin $85 \mathrm{mg} \mathrm{m}^{-2}$ on day 2 , with cycles repeated every 3 weeks.

\section{PATIENTS AND METHODS}

\section{Patient selection}

Patients with gastric or GEJ adenocarcinoma with distant metastasis not previously treated by systemic chemotherapy were enrolled onto the study. Adjuvant chemotherapy without agents under the study was allowed if completed at least 6 months before. The patients were required to have measurable disease, ECOG 
performance status $\leqslant 2$, life expectancy $>3$ months, age between 18 and 75 years, adequate bone marrow (absolute neutrophil count $\geqslant 1500 \mu \mathrm{l}^{-1}$, platelet count $\geqslant 100000 \mu \mathrm{l}^{-1}$ ), renal (serum creatinine $\leqslant 1.5 \mathrm{mg} \mathrm{dl}^{-1}$ ) and liver (serum bilirubin $\leqslant 1.5 \mathrm{mg} \mathrm{dl}^{-1}$ ) functions, normal cardiac function, absence of second primary tumour other than non-melanoma skin cancer or in situ cervical carcinoma, no CNS involvement, no previous radiotherapy in parameter lesions, no concurrent uncontrolled medical illness. The protocol was approved and carried out according to the principles of the Declaration of Helsinki and Good Clinical Practice Guidelines, and all patients gave their written informed consent to participate onto the trial.

\section{Treatment}

Treatment consisted of irinotecan $150 \mathrm{mg} \mathrm{m}^{-2}$ diluted in $250 \mathrm{ml}$ of normal saline as a $1 \mathrm{~h}$ infusion followed by docetaxel $60 \mathrm{mg} \mathrm{m}^{-2}$ diluted in $500 \mathrm{ml}$ of normal saline as $1 \mathrm{~h}$ infusion on day 1 , and oxaliplatin $85 \mathrm{mg} \mathrm{m}^{-2}$ diluted in $500 \mathrm{ml} 5 \%$ dextrose as a $2 \mathrm{~h}$ infusion on day 2. Cycles were repeated every 3 weeks. Antiemetic treatment consisted of an antiserotonin agent plus dexamethasone in a $15 \mathrm{~min}$ infusion before starting chemotherapy. In addition, orally prednisone premedication was used for prophylaxis of docetaxel-induced hypersensitivity and fluid retention. Atropine $0.25 \mathrm{mg}$ subcutaneously was administered for prophylaxis of cholinergic syndrome. Granulocyte colony-stimulating factor (G-CSF) was used only as secondary prophylaxis once patients had febrile neutropaenia or documented neutropaenic infection. Treatment was postponed by a maximum of 2 weeks if the absolute neutrophil count was less than $1500 \mu \mathrm{l}^{-1}$ or the platelet count was less than $100000 \mu \mathrm{l}^{-1}$. The dose of irinotecan was reduced by $20 \%$ of the previous dose in case of grade $\geqslant 3$ diarrhoea, whereas oxaliplatin was reduced by $25 \%$ in case of grade $\geqslant 2$ peripheral neuropathy, and docetaxel by $25 \%$ in case of the following toxicities: grade $\geqslant 3$ neutropaenia lasting more than 7 days (or in presence of fever), second incidence of febrile neutropaenia despite G-CSF support administered after the first occurrence, grade $\geqslant 3$ diarrhoea and grade $\geqslant 3$ stomatitis.

Chemotherapy was generally administered on an outpatient basis for a maximum of eight cycles and was discontinued in case of unacceptable toxicity, treatment delay longer than 2 weeks, disease progression or patients' refusal.

\section{Pretreatment and follow-up studies}

Pretreatment evaluation included clinical history and physical examination, automated blood cell count, biochemical profile, ECG and computed tomography of thorax and abdomen. Endoscopy was performed only in case of complete remission of all measurable lesions. Blood counts were obtained weekly; biochemical profile was repeated every 3 weeks. All measurable parameters of disease were re-evaluated every 6 weeks, until the tumour progressed.

\section{Evaluation of response and toxicity}

Patients were evaluated for response to chemotherapy every two cycles of treatment. Responses were assessed by at least two observers, and were confirmed by an expert independent radiologist. The RECIST criteria were used to evaluate clinical response (Therasse et al, 2000), and all objective responses were confirmed by CT scans at least 4 weeks after the initial documentation of response. Time-to-progression and OS were calculated from the date of first chemotherapy cycle to the date of disease progression, death or last follow-up evaluation, respectively. Toxicity was assessed in each treatment cycle using the National Cancer Institute Common Toxicity Criteria (version 3.0).

\section{Statistical considerations}

The primary end point of this study was to test the hypothesis that TTP will improve by $50 \%$. The major secondary end point was RR. Other secondary end points were OS and safety. The study was designed to have an $80 \%$ power to show an improvement in median TTP from 4 to 6 months with a 5\% type I error, two sides, assuming exponential progression-free survival times. A sample size of 40 patients was required to meet these requirements (A'Hern, 2001). Time-to-progression and OS were analysed according to the Kaplan-Meier method, and were updated to 30 April 2007.

\section{RESULTS}

\section{Patients' characteristics}

From February 2004 to April 2006, 40 patients with metastatic gastric or GEJ cancer entered onto this multicentre study. All patients were evaluable for efficacy and toxicity. The pretreatment characteristics of patients are listed in Table 1. None of the patients had previously received chemotherapy for advanced disease; six patients had received adjuvant chemotherapy without irinotecan, docetaxel or oxaliplatin several months before they entered this study (median, 14 months; range, 9-23 months).

\section{Efficacy}

Median TTP was 6.5 months (95\% confidence interval (CI) 5.67.4) (Figure 1). Only six patients progressed within the first 2 months, whereas at the time of this analysis all but one patient progressed. Among 40 assessable patients, we observed two (5\%) complete responses (CRs) and $18(45 \%)$ partial responses (PRs), for an overall RR of $50 \%$ (95\% CI 35-65\%). Disease remained stable in $14(35 \%)$ patients (Table 2). Responses according to predominant site of disease, were as follows: liver 16 of 28 patients (57\%); nodes/peritoneum 3 of 10 patients (30\%); lung 1 of 2 patients. RRs did not significantly differ according to number of metastatic sites: one site, 7 of 12 patients (58\%); two sites, 9 of 18 patients $(50 \%)$; and three or more sites, 4 of 10 patients $(40 \%)$. Responses were seen in 3 of 6 patients $(50 \%)$ who received adjuvant chemotherapy and in 17 of 34 patients (50\%) not previously treated with chemotherapy. Responses were observed also in 13 of 26 patients (50\%) with primary tumour not resected and in 7 of 14 patients $(50 \%)$ with primary tumour resected. Response rates and TTP did not differ when patients were evaluated according to the primary site of disease (gastric: $50 \%$ and 6.5 months; GEJ: $50 \%$ and 6.3 months, respectively). Upon disease progression, 21 patients $(52.5 \%)$ received a second-line chemotherapy, including epirubicin/fluorouracil $(n=16)$ and cisplatin/capecitabine $(n=5)$. Median OS was 11.5 months $(95 \%$ CI 8.7-14.3 months) (Figure 2). One- and two-year survivals were 42 and $15.8 \%$, respectively. Thirty-eight patients had died at the time of the present evaluation. Twenty-three of the 32 patients $(72 \%)$ who had tumour-related symptoms before therapy showed an improvement in at least one of their symptoms without worsening of any other symptom.

\section{Toxicity}

Haematological toxicity data are listed in Table 3. A total of 242 cycles of IDO regimen were analysed in 40 patients, with a median of seven cycles administered per patient (range, 1-8 cycles). The most important toxicity was myelosuppression, which occurred almost always on day 8 (docetaxel nadir). Grade 3 and 4 neutropaenia were recorded in 40 and in $7.5 \%$ of the patients, respectively. Four episodes of febrile neutropaenia occurred in 3 
Table I Patient's characteristics

\begin{tabular}{|c|c|c|}
\hline Characteristic & No. of patients & $\%$ \\
\hline Patients evaluable & 40 & 100 \\
\hline \multicolumn{3}{|l|}{ Age (years) } \\
\hline Median & 62 & \\
\hline Range & $38-75$ & \\
\hline \multicolumn{3}{|l|}{ Sex } \\
\hline Male & 28 & 70 \\
\hline Female & 12 & 30 \\
\hline \multicolumn{3}{|l|}{ ECOG PS } \\
\hline 0 & 8 & 20 \\
\hline I & 26 & 65 \\
\hline 2 & 6 & 15 \\
\hline \multicolumn{3}{|l|}{ Disease location } \\
\hline Gastric & 30 & 75 \\
\hline GEJ & 10 & 25 \\
\hline \multicolumn{3}{|l|}{ Histologic type } \\
\hline Diffuse & 18 & 45 \\
\hline Intestinal & 16 & 40 \\
\hline Unspecified & 6 & 15 \\
\hline Previous adjuvant chemotherapy & 6 & 15 \\
\hline \multicolumn{3}{|l|}{ Status of primary tumour } \\
\hline Unresected & 26 & 65 \\
\hline Resected & 14 & 35 \\
\hline \multicolumn{3}{|l|}{ Predominat site of disease } \\
\hline Liver & 28 & 70 \\
\hline Nodes/peritoneum & 10 & 25 \\
\hline Lung & 2 & 5 \\
\hline \multicolumn{3}{|l|}{ No. of metastatic sites } \\
\hline 1 & 12 & 30 \\
\hline 2 & 18 & 45 \\
\hline$\geqslant 3$ & 10 & 25 \\
\hline \multicolumn{3}{|l|}{ Symptoms } \\
\hline \multicolumn{3}{|l|}{ Weight loss } \\
\hline No & 13 & 32.5 \\
\hline$\leqslant 10 \%$ & 16 & 40 \\
\hline$>10 \%$ & 11 & 27.5 \\
\hline \multicolumn{3}{|l|}{ Anorexia } \\
\hline No & 21 & 52.5 \\
\hline Yes & 19 & 47.5 \\
\hline \multicolumn{3}{|l|}{ Dysphagia } \\
\hline No & 23 & 57.5 \\
\hline Yes & 17 & 42.5 \\
\hline \multicolumn{3}{|l|}{ Pain } \\
\hline No & 20 & 50 \\
\hline Yes & 20 & 50 \\
\hline
\end{tabular}

Abbreviations: ECOG PS=Eastern Cooperative Oncology Group Performance Status; GEJ = gastroesophageal junction.

(7.5\%) patients. In these patients, a $25 \%$ dose reduction of docetaxel was required, whereas treatment was postponed in two (5\%) patients and in six $(2.5 \%)$ cycles because of a delay in bone marrow recovery. Mean irinotecan, docetaxel and oxaliplatin dose intensities were $45.45,18.36$ and $27.17 \mathrm{mg} \mathrm{m}^{-2}$ per week, respectively, which are equivalent at $90.9,91.8$ and $95.9 \%$ of the planned dose intensities for these drugs. Grade 3 thrombocytopaenia was observed in $2.5 \%$ of the patients, and grade 3 anaemia occurred in $10 \%$ of the patients.

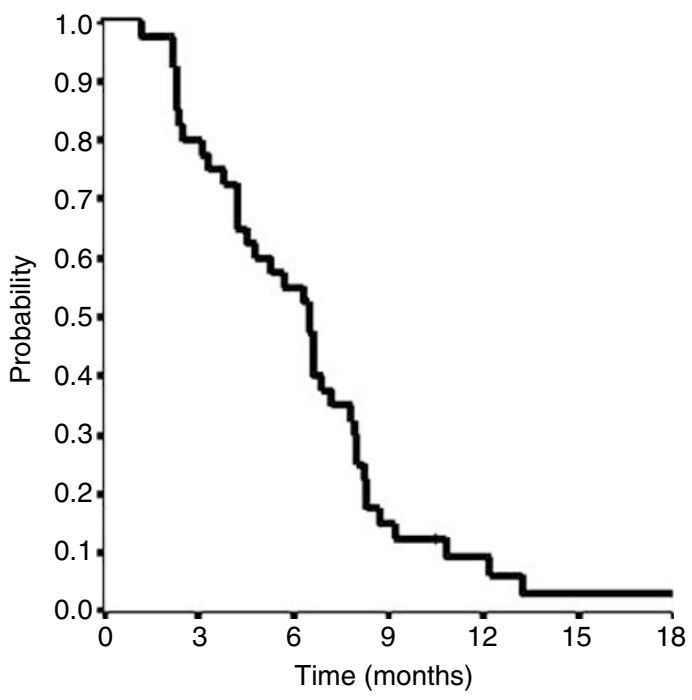

Figure I Median TTP for all patients.

Table 2 Objective response in 40 patients

\begin{tabular}{lcr}
\hline Response & No. of patients & $\%$ \\
\hline Complete response & 2 & 5 \\
Partial response & 18 & 45 \\
Stable disease & 14 & 35 \\
Progressive disease & 6 & 15 \\
\hline
\end{tabular}

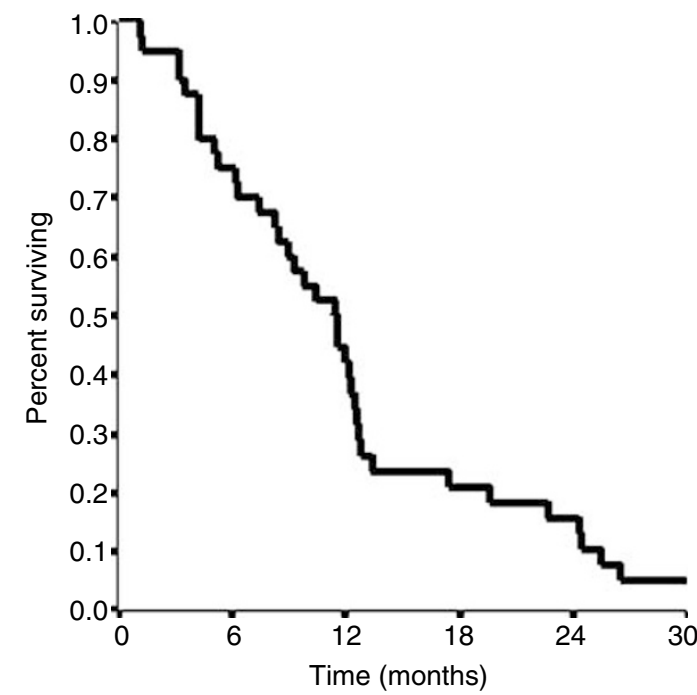

Figure 2 Overall survival for all patients.

Non-haematological toxicities are listed in Table 4. Mild-tomoderate diarrhoea, which was reversible and manageable in all cases, occurred in $40 \%$ of the patients, while grade 3 diarrhoea developed in four (10\%) patients. Alopecia was frequent. Mild nausea and vomiting was encountered in $45 \%$ of the patients, and was severe in three $(7.5 \%)$ patients. Mild and transient peripheral neurotoxicity was recorded in $35 \%$ of the patients. Hypersensitivity reactions, which not precluded chemotherapy continuation, were recorded in $5 \%$ of the patients. No cardiotoxicity or treatment-related deaths were observed. 
Table 3 Grade 3/4 haematological toxicity per cycle and per patient

\begin{tabular}{lcccccc}
\hline & \multicolumn{2}{c}{$\%$ of $\mathbf{2 4 2}$ cycles } & & \multicolumn{2}{c}{ \% of $\mathbf{4 0}$ patients } \\
\cline { 2 - 3 } \cline { 6 - 7 } Toxicity & Grade $\mathbf{3}$ & Grade & & Grade 3 & Grade 4 \\
\hline Neutropaenia & 26 & 5 & & 40 & 7.5 \\
Thrombocytopaenia & 1 & - & & 2.5 & - \\
Anaemia & 4.5 & - & & 10 & - \\
\hline
\end{tabular}

Four episodes of febrile neutropaenia in three patients.

Table 4 Non-haematological toxicity in 40 patients

\begin{tabular}{lccc}
\hline Toxicity & Grade I \% & Grade 2 \% & Grade 3 \% \\
\hline Nausea/vomiting & 25 & 20 & 7.5 \\
Mucositis & 20 & 15 & 5 \\
Diarrhoea & 20 & 20 & 10 \\
Fatigue & 25 & 15 & 7.5 \\
Fluid retention & 15 & 10 & - \\
Alopecia $_{\text {Neurotoxicity }}$ & 30 & 45 & 25 \\
Hypersensitivity reaction $^{2}$ & 20 & 15 & - \\
\hline
\end{tabular}

${ }^{\mathrm{a}}$ Grade $\mathrm{I}-2=$ mild; grade $3=$ severe.

\section{DISCUSSION}

Classical cisplatin-based regimens obtain responses in $20-40 \%$ of the patients with metastatic gastric or GEJ cancer, a median TTP of about 4 months and a median survival of 8-9 months (Wagner et al, 2006). Despite recent advances in the chemotherapy of this disease, the survival advantage appears to be marginal, and no regimens have emerged that could be considered standard.

To develop a potentially more effective front-line chemotherapy, combination regimens have generally included one or two of the three new drugs active in gastric cancer, namely irinotecan, docetaxel and oxaliplatin. This is the first study that combines these three agents.

The IDO regimen appears to be an active treatment in previously untreated patients with metastatic gastric cancer, since the primary end point of this study was largely achieved with a median TTP of 6.5 months. Noteworthy are also the overall RR of $50 \%$ and the median survival of 11.5 months. These data appear of particular importance considering that liver involvement and primary tumour unresected were recorded in 70 and $65 \%$ of the patients, respectively.

These results compare favourably with those reported in several trials using new generation treatment regimens. In a recent phase III randomised trial, the combination of docetaxel, cisplatin and fluorouracil demonstrated significant superiority in comparison to the reference regimen of cisplatin and fluorouracil (CF). Nevertheless, TTP, OS and RR were 5.6 months, 9.2 months and 37\%, respectively (Van Cutsem et al, 2006). In another phase III study of
337 patients with advanced gastric cancer, irinotecan in combination with bolus fluorouracil and infused fluorouracil over $22 \mathrm{~h}$ did not result in statistically significant improvement in TTP (5.0 vs 4.2 months) or OS (9.0 vs 8.7 months) compared with CF, but showed a better safety profile (Dank et al, 2005). Moreover, our results are similar to those observed with the combination of EOX in the REAL-2 phase III study. In this trial, the EOX regimen showed RR of $48 \%$ and OS of 11.2 months in 239 patients with advanced oesophageal, EGJ or gastric carcinoma with a manageable toxicity (Cunningham et al, 2006).

The combination used in the current study compares favourably also with regimens including two of these three new agents. In two phase II studies in advanced gastric cancer, combined irinotecan and oxaliplatin yielded a median TTP of 5.5 and 5.3 months, and an OS of 8.5 and 9.5 months (Souglakos et al, 2004; Woell et al, 2006). When irinotecan was used in combination with docetaxel in 90 untreated patients with advanced gastric cancer enrolled in two phase II studies, a median TTP of 3.8 and 4.5 months with an OS of 8.2 and 9.0 months were observed (Hawkins et al, 2003; Park et al, 2006). Treatment results do not seem to improve by combining oxaliplatin and docetaxel, since TTP and OS were 4.4 and 9.5 months, respectively, in 71 patients with stage IV gastroesophageal and/or stomach cancer (Richards et al, 2006).

Of considerable importance in patients with metastatic gastric cancer are treatment tolerance and quality-of-life, since these patients have generally symptoms at baseline. The IDO regimen demonstrated a tolerable toxicity profile in the present trial. The most important side effect was grade 3-4 neutropaenia, which occurred in less than $50 \%$ of the patients, and almost always at docetaxel nadir. Grade 3 diarrhoea was observed in four patients $(10 \%)$. Other severe toxic effects were infrequent, as were dose reductions and treatment delays. Although quality-of-life was not measured in our study, the majority of patients enjoyed a clear clinical benefit during treatment, as evidenced by the relief of pain and other tumour-related symptoms. Treatment compliance was good, as demonstrated by relative dose intensities of $90.9 \%$ for irinotecan, $91.8 \%$ for docetaxel and $95.8 \%$ for oxaliplatin. Moreover, chemotherapy was generally administered on an outpatient basis.

In conclusion, we found that the combination of irinotecan, docetaxel and oxaliplatin is active with an acceptable safety profile in the first-line treatment of metastatic gastric or GEJ adenocarcinoma, and should be compared with other active regimens (e.g. ECF and EOX) in a randomised fashion. However, in order to further improve treatment results in this disease, the addition of targeted therapy to cytotoxic agents should be considered. In two recent phase II trials, the combination of irinotecan, cisplatin and bevacizumab (Shah et al, 2006), and cetuximab combined with FOLFIRI (Pinto et al, 2007), both produced encouraging results with TTP and OS remarkably improved over historical controls. In this context, the IDO regimen, with its activity and safety profile, could be a good candidate to enhance survival in metastatic gastric or GEJ cancer patients.

\section{REFERENCES}

A'Hern RP (2001) Sample size tables for exact single-stage phase II designs. Statist Med 20: 859-866

Ajani J (2006) Review of capecitabine as oral treatment of gastric, gastroesophageal, and esophageal cancers. Cancer 107: $221-231$

Al-Batran SE, Atmaca A, Hegewisch-Becker S, Jaeger D, Hahnfeld S, Rummel MJ, Seipelt G, Rost A, Orth J, Knuth A, Jaeger E (2004) Phase II trial of biweekly infusional fluorouracil, folinic acid, and oxaliplatin in patients with advanced gastric cancer. J Clin Oncol 22: 658-663

Bleiberg H (1999) CPT-11 in gastrointestinal cancer. Eur J Cancer 35: $371-379$

Chang HM, Kim TW, Yook JH, Oh ST, Kim WK, Lee JS, Kang JK (2003) Phase II study of irinotecan (I) and docetaxel (D) as second line chemotherapy for patients with previously treated advanced gastric cancer (AGC). Proc Am Soc Clin Oncol 22: 328 (abstract 1316)

Cunningham D, Rao S, Starling $\mathrm{N}$, Iveson $\mathrm{T}$, Nicolson $\mathrm{M}$, Coxon $\mathrm{F}$, Middleton G, Daniel F, Oates J, Norman AR (2006) Randomized multicentre phase III study comparing capecitabine with fluorouracil and oxaliplatin with cisplatin in patients with advanced oesophagogastric (OC) cancer: The REAL 2 trial. J Clin Oncol 24(S18): 182s (abstract LBA4017) 
Dank M, Zaluski J, Barone C, Valvere V, Peschel C, Wenczl M, Goker E, Risse M, Award L, Bugatet R (2005) Randomized phase 3 trial of irinotecan (CPT-11) + 5FU/folinic acid (FA) vs CDDP+5FU in 1st-line advanced gastric cancer patients. J Clin Oncol 23(S16): 308s (abstract 4003)

Devesa SS, Fraumeni JF (1999) The rising incidence of gastric cardia cancer. J Natl Cancer Inst 91: 747-749

Hawkins R, Cunningham D, Soerbye H, Adenis A, Canon JL, LopezVivanco G, Jacques C, Stenger P, Zuber E, Misset JL (2003) Randomized phase II trial of docetaxel plus irinotecan $v s$ docetaxel plus 5-fluorouracil $(5 \mathrm{FU})$ in patients with untreated advanced gastric adenocarcinoma (AGAC). Proc Am Soc Clin Oncol 22: 257 (abstract 1032)

Hundahl S, Phillips J, Menck H (2000) The national cancer data base report on poor survival of US gastric carcinoma patients treated with gastrectomy: fifth edition American joint committee on cancer staging, proximal disease, and the ' different disease' hypothesis. Cancer 88: $921-932$

Kamangar F, Dores GM, Anderson WF (2006) Patterns of cancer incidence, mortality, and prevalence across five continents: defining priorities to reduce cancer disparities in different geographic regions of the world. J Clin Oncol 24: 2137-2150

Kohne CH, Thuss-Patience P, Catane R, Klein H, Peretz T, Preusser P, Niederle N, Ducreux M, Hansjokem W, Jacques C (1999) Final results of a phase II trial of CPT-11 in patients with advanced gastric cancer. Proc Am Soc Clin Oncol 18: 258 (abstract 993)

Louvet C, André T, Tigaud JM, Gamelin E, Douillard JY, Brunet R, Francois E, Jacob JH, Levoir D, Taamma A, Rougier P, Cvitkovic E, de Gramont A (2002) Phase II study of oxaliplatin, fluorouracil, and folinic acid in locally advanced or metastatic gastric cancer patients. J Clin Oncol 20: $4543-4548$

Park SR, Chun JH, Yu MS, Lee JH, Ryu KW, Choi IJ, Kim CG, Lee JS, Kim YW, Bae JM, Kim HK (2006) Phase II study of docetaxel and irinotecan combination chemotherapy in metastatic gastric carcinoma. Br J Cancer 94: $1402-1406$

Pinto C, Di Fabio F, Siena S, Cascinu S, Rojas Llimpe FL, Ceccarelli C, Mutri V, Giannetta L, Giaquinta S, Funaioli C, Berardi R, Longobardi C, Piana E, Martoni A (2007) Phase II study of cetuximab in combination with
FOLFIRI in patients with untreated advanced gastric or gastroesophageal junction adenocarcinoma (FOLCETUX study). Ann Oncol 18: 510-517

Richards DA, Wilfong L, Reznick D, McCollum D, Boehm KA, Zhan L, Asmar L (2006) Phase II multicenter trial of docetaxel+oxaliplatin in stage IV gastroesophageal and/or stomach cancer. J Clin Oncol 24(S18): 195s (abstract 4071)

Rivera F, Vega-Villegas ME, Lopez-Brea MF (2007) Chemotherapy of advanced gastric cancer. Cancer Treat Rev 33: 315-324

Shah MA, Ramanathan RK, Ilson DH, Levnor A, D'Adamo D, O’Reilly E, Tse A, Trocola R, Schwartz L, Capanu M, Schwartz GK, Kelsen DP (2006) Multicenter phase II study of irinotecan, cisplatin, and bevacizumab in patients with metastatic gastric or gastroesophageal junction adenocarcinoma. J Clin Oncol 24: 5201-5206

Souglakos J, Syrigos K, Potamianou A, Polyzos A, Boukovinas I, Androulakis N, Kouroussis C, Vardakis N, Christophilakis C, Kotsakis A, Georgoulias V (2004) Combination of irinotecan (CPT-11) plus oxaliplatin (L-OHP) as first-line treatment in locally advanced or metastatic gastric cancer: a multicentre phase II trial. Ann Oncol 15: $1204-1209$

Therasse P, Arbuck SG, Eisenhauer E, Wanders J, Kaplan RS, Rubinstein L, Verweij J, Van Glabbeke M, van Oosterom AT, Christian MC, Gwyther SG (2000) New guidelines to evaluate the response to treatment in solid tumours. J Natl Cancer Inst 92: 205-216

Thuss-Patience PC, Kretzschmar A, Reichardt P (2006) Docetaxel in the treatment of gastric cancer. Future Oncol 2: 603-620

Van Cutsem E, Moiseyenko VM, Tjulandin S, Majlis A, Constenla M, Boni C, Rodigues A, Fodor M, Chao Y, Voznyi E, Risse ML, Ajani JA (2006) Phase III study of docetaxel and cisplatin plus fluorouracil compared with cisplatin and fluorouracil as first-line therapy for advanced gastric cancer: a report of the V325 study group. J Clin Oncol 24: 4991-4997

Wagner AD, Grothe W, Haerting J, Kleber G, Grothey A, Fleig WE (2006) Chemotherapy in advanced gastric cancer: a systemic review and metaanalysis based on aggregate data. J Clin Oncol 24: 2903-2909

Woell E, Kühr T, Eisterer W, Gattringer K, Greil R, Zabernigg A, Hilbe W, Thaler J (2006) Oxaliplatin and irinotecan chemotherapy in advanced gastric cancer. Final results of a multicenter phase II trial. J Clin Oncol 24(S18): 195s (abstract 4070) 